\title{
Paternal Relative
}

National Cancer Institute

\section{Source}

National Cancer Institute. Paternal Relative. NCI Thesaurus. Code C71382.

A person related by lineage through the father's side of the family. 